\title{
Energy Aware Coverage Preservation and Lifetime Enhancement Protocol for Wireless Sensor Network
}

\author{
Arti Rana \\ M.Tech CSE \\ Uttarakhand Technical \\ University \\ Dehradun, India
}

\author{
Anshika Garg \\ Assistant Professor \\ Uttarakhand Technical \\ University \\ Dehradun, India
}

\author{
Vivek Kumar \\ M.Tech CSE \\ Uttarakhand Technical \\ University \\ Dehradun, India
}

\begin{abstract}
A wireless sensor network consists of spatially distributed autonomous sensors to monitor physical and environmental conditions. One of the major concerns in wireless sensor network is increasing an energy-efficient protocol which has an important impact on the overall network lifetime of the sensor network.

In this work, we propose a new clustering scheme called Energy-Aware Coverage Preservation and Lifetime Enhancement Protocol for Wireless Sensor Network (EACPLEP) which is essentially a variation of an existing routing scheme, An Enhanced Energy-Efficient Protocol with Static Clustering ( $\left.E^{3} \mathrm{PSC}\right)$. Alike to $\mathrm{E}^{3} \mathrm{PSC}$, the presented work partitions the network into distance-based static clusters, reduce the overhead of dynamic clustering. However, disparate $\mathrm{E}^{3} \mathrm{PSC}$, the selection of cluster heads are based on density aware scheme in which, where the number of nodes are more, the cluster head formed on that side. Performance of the scheme is considered in terms of network lifetime. A set of experimentation is carried out to assess the performance of the scheme and to compare the results with $\mathrm{E}^{3} \mathrm{PSC}$. Based on our experimental results, it has been found that EACPLEP outperforms $\mathrm{E}^{3} \mathrm{PSC}$ in terms of network lifetime and energy consumption.
\end{abstract}

\section{Keywords}

Cluster head, Energy-Efficiency, Network Lifetime, and Wireless Sensor Network.

\section{INTRODUCTION}

A wireless sensor network is a collection of sensor nodes interconnected by wireless communication channels. Each sensor node is a small device that can collect data from its surrounding area, carry out simple computations, and communicate with other sensors or with the base stations (BS). Such networks have been realized due to recent advances in micro electromechanical systems and are expected to be widely used for applications such as environment monitoring, home security, and earthquake warning [1].

The advent of efficient short range radio communication and advances in miniaturization of computing devices have given rise to strong interest in wireless sensor networks [2], [3]. A wireless sensor network (WSN) consists of hundreds or thousands of MEMS-based sensor nodes with the station either directly (single hop) or via other nodes (multi hop) around it in a cooperative manner. Such as network typically suffers from a number of unavoidable problems, such as resource-constrained nodes, random node deployment sometimes in an unattended open field etc. In some critical applications e.g. medical instrument monitoring it is very difficult to replace/recharge battery. Therefore, the network as a whole must minimize the energy usage in order to enable untethered and unattended operation for an extended period of time.

Many works are so far reported towards minimization of energy usage. One of the ways to minimize such energy usage is employment of clustering. Clustering is defined [4] as the grouping of similar objects or the process of finding a natural association among some specific objects or data. It is used in WSN to transmit processed data to base station minimizing the number of nodes that take part in long distance communication leading to lowering of total energy consumption of the system.

A number of works related to energy saving approaches exploiting cluster-based data gathering in WSNs have been defined in literature [5-10].

In this present work, we propose an EACPLEP (EnergyAware Coverage Preservation and Lifetime Enhancement Protocol), a hierarchical static clustering based protocol, which eliminates the overhead of dynamic clustering and engages high power sensor nodes for power consuming tasks and as a result prolongs the network lifetime. In each cluster, EACPLEP chooses the sensor node with maximum energy as the cluster-head $(\mathrm{CH})$ on the basis of density in which where the number of nodes are maximum, cluster head is formed on that side; thus not only there is always one $\mathrm{CH}$ for each cluster, but also the overhead of dynamic clustering is removed. The rest of the paper is organized as follows. Section 2 describes the proposed scheme along with the equivalent algorithm. Performance -assessment and simulation results are obtained in section 3 . The entire work is concluded in section 4 .

\section{PROPOSED SCHEME}

This section includes the proposed scheme, Energy-Aware Coverage Preservation and Lifetime Enhancement Protocol (EACPLEP) followed by the algorithm of the proposed scheme. The objective of the scheme is to develop an energyefficient clustering in wireless sensor networks to achieve the desired features of prolonged network lifetime and coveragepreservation and to select temporary cluster-heads and clusterheads for the clusters so that all the clusters remain energy balanced resulting in prolonged network lifetime.

\subsection{Network Model}

We consider a WSN with the following properties [5]-[6], [9][10];

- All sensor nodes are immobile and homogenous in terms of initial stored energy. 
- The nodes are equipped with power control capabilities to vary their transmitted power.

- Network adopts continuous data flow model.

- Base station is fixed and not located among the sensor nodes.

- Base station knows locations of all the sensor nodes deployed in the network.

\subsection{Protocol Architecture}

Like $\mathrm{E}^{3} \mathrm{PSC}$ [11], EACPLEP is also a self-organizing, static clustering system in which clusters are produced only once during the network action. The whole network -operation is invented to be divided into a number of rounds where each round is divided into three phases- setup phase, responsible node selection phase, and the steady-state phase. The extended descriptions of these phases are as follows.

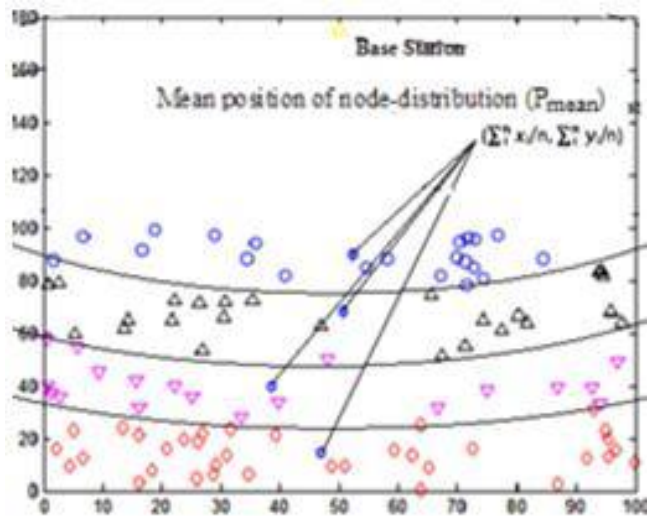

Figure 1. Distance based cluster formation showing mean position of node distribution for each cluster

1) Set-up Phase: According to the static clustering method which is used in $\mathrm{E}^{3} \mathrm{PSC}$, foundation of cluster is performed only once at the beginning of network operation. Firstly, the sink broadcast (k-1) particular messages with different transmission powers, where $\mathrm{k}$ denotes the desired number of clusters to be determined by base station a priori. By broadcasting $\mathrm{k}=1$ message, all the sensor nodes which are in the range of this message, have the sense of hearing this message set their cluster ID to $\mathrm{k}$ and inform the base station that they are the parts of the cluster ' $\mathrm{k}$ ' via spreading a join-request message (Join-REQ) backside to the base-station. Correspondingly, by broadcasting the $\mathrm{k}=\mathrm{k}-1$ message, all the sensor nodes which are not joined to any clusters up till now and listen this message set their cluster ID to k-1 and report to base station with a Join-REQ message. Afterward, all sensor nodes which are not joined to any clusters set their cluster ID to $k$ and inform base station.

Once the clusters are produced, base station computes the mean position of node-distribution $\left(\mathrm{P}_{\text {mean }}\right.$ ) of each cluster where $i$ is cluster ID. Succeeding to this, for all the nodes in a cluster $r_{i}$, the base station computes distance $\mathbf{d}_{\text {mean }}{ }^{\mathbf{j}}{ }_{i}$ from $\mathrm{P}_{\text {mean } i}$ where $\boldsymbol{j}$ is the node ID within cluster $\boldsymbol{i}$. Beside these estimations, base station selects a temporary clusterhead $\left(\mathrm{TCH}_{\mathrm{i}}\right)$ for every cluster in a arbitrary method and arranges a TDMA program for the nodes in each cluster of the network. After performing these tasks, base station broadcast the 3-tuple data $\boldsymbol{( \boldsymbol { T C H }}$,
TDMA - schedule ${ }_{i}{ }_{i}, \boldsymbol{d}_{\text {mean }} \mathbf{j}_{\mathrm{i}}$ ) to the nodes in each cluster. Once all the nodes in the cluster receive the 3-tuple data, set-up phase is complete.

2) Responsible Node Selection Phase: In this phase, firstly, take temporary-cluster-heads (TCHs) as a reference node. After choosing TCHs, now divide the clusters into two groups, i.e. Right Group and Left Group. Later than partition of clusters, now count the total energy of every group called as $\mathrm{R}_{\text {ENERGY }}$ and $\mathrm{L}_{\text {ENERGY. If }} \mathrm{R}_{\text {ENERGY }}>\mathrm{L}_{\text {ENERGY }}$ then select the node with highest energy from Right Group otherwise select the node with highest energy from Left Group.

3) Steady-State Phase: In this phase, alike to $\mathrm{E}^{3} \mathrm{PSC}$ [11], nodes send their sensed records to related cluster head during their pre-allocated fixed time period representing the detail that the time required for sending a frame depends upon the amount of nodes in the cluster. In a cluster, radios of nodes are kept off until their allocated time periods come but radio of cluster-head is kept on always to receive records or data from all the nodes. Moreover, $\mathrm{CHs}$ and base station converse subsequent direct transmission. After a predefined time-period, a round terminates.

\subsection{Algorithm (EACPLEP)}

1. BEGIN

$1 *$ Setup Phase ( jobs are executed by the sink) */

2 . Form $\boldsymbol{k}$ clusters based on the density of nodes

3. For $\mathrm{i} \leftarrow 1$ to $\mathrm{k}$

4. Calculate mean position of node allocation, $\mathrm{P}_{\text {mean }}$ ${ }_{i}$ in cluster ${ }_{i}$

5 . For $\mathrm{j} \leftarrow 1$ to $\mathrm{mi} / * \mathrm{mi} \rightarrow$ No. of nodes in cluster *1

6. Compute distance of $\boldsymbol{n o d e}_{\boldsymbol{j}}$ from $\mathrm{P}_{\text {mean } i}, \boldsymbol{d}_{\text {mean } \boldsymbol{i}} \boldsymbol{j}$

7. End For

8. Select Temporary Cluster Head (TCHi) at random for cluster $_{i}$

9. Generate TDMA schedule for the nodes of cluster $_{i}$

10. For $\mathrm{j} \leftarrow 1$ to $\mathrm{m}_{\mathrm{i}}$

11. Send 3-tuple $\left(\boldsymbol{T C H} \boldsymbol{H}_{i}, \boldsymbol{T D M A} \boldsymbol{A}_{i}, \boldsymbol{d m e a n}_{\boldsymbol{i}}{ }^{j}\right)$ data to node $_{j}$

12. End For

13. End For

/* End of Setup Phase */

14 . For $\mathrm{v} \leftarrow 1$ to round

$/ *$ round $\rightarrow$ Total no. of rounds

/* Responsible Node Selection Phase */

15. For $\mathrm{i} \leftarrow 1$ to $\mathrm{k} / *$ for every cluster $\mathrm{i}^{*} /$

16. For $\mathrm{j} \leftarrow 1$ to $\boldsymbol{m}_{\boldsymbol{i}} / *$ Alive nodes*/

17. Divide clusters into two group /* i.e. Left group and Right group*/

18.Count the total energy of every group $/ *$ i.e.

$\mathrm{R}_{\text {energy }}$ and $\mathrm{L}_{\text {energy }} * /$

19. if $\mathrm{R}_{\text {energy } i}{ }^{j}>\mathrm{L}_{\text {energy } i}{ }^{j}$

20. send $\left(\mathrm{R}_{\text {energy } i}{ }^{j}\right)$ to $\mathrm{BS}$

21. end if

22. End For

/* $\mathrm{TCH}_{i}$ performs the following tasks $* /$

23. Call Responsible_Node_Selection()

/* Select $\boldsymbol{C H i}$ for the current round

Select $\boldsymbol{T C H} \boldsymbol{i}$ for the next round */

24. End For 


\author{
/* Steady-State Phase */ \\ 25 . For $\mathrm{i} \leftarrow 1$ to $\mathrm{k}$ \\ 26. For Alive_node $\leftarrow 1$ to $\boldsymbol{m i}$ \\ 27. Send data to $\boldsymbol{C H}$ \\ /*Data transmission by Alive_node(s) */ \\ 28. End For \\ 29. Send aggregated data to the base station \\ /* Data transmission by $\mathbf{C H i} *$ / \\ 30. End For \\ 31. End For $/ *$ End of rounds */ \\ 32. END /* End of Algorithm */
}

\section{PERFORMANCE EVALUATION}

\subsection{Quantitative Analysis}

\subsubsection{Simulation Environment:}

To evaluate the performance of EACPLEP, MATLAB 7.1 is used as a simulation tool. We consider that the sensor nodes are positioned randomly across in plain area of $\left(\mathrm{x}_{\mathrm{m}}=100 \mathrm{~m} \mathrm{X}\right.$ $\left.\mathrm{y}_{\mathrm{m}}=100 \mathrm{~m}\right)$ and the base station is located at $\left(\mathrm{x}=0.5^{*} \mathrm{x}_{\mathrm{m}}\right.$, $\left.\mathrm{y}=\mathrm{y}_{\mathrm{m}}+75\right)$ i.e. $(\mathrm{x}=50, \mathrm{y}=175)$. Each node is equipped with equal amount of initial energy (2J) at the beginning of the simulation. Further we assume that WSN is working in continuous data flow application domain. Table 1 represents various parameters and their values used in simulation.

\section{TABLE 1. PARAMETERS AND CORRESPONDING} VALUES USED IN SIMULATION

\begin{tabular}{|c|c|}
\hline Parameter & Parameter's Name \\
\hline Network Area & $100 \mathrm{~m} \mathrm{X} 100 \mathrm{~m}$ \\
\hline Base Station's Position & $(50 \mathrm{~m}, 175 \mathrm{~m})$ \\
\hline Initial Energy for Nodes & 2 Joule \\
\hline Number of Deployed Nodes & 100 \\
\hline Size of Data Message & $4000 \mathrm{bits}$ \\
\hline $\begin{array}{c}\text { Energy Consumed in Data } \\
\text { Aggregation(E }\left(\mathrm{E}_{\mathrm{DA}}\right)\end{array}$ & $5 \mathrm{~nJ} / \mathrm{bit} / \mathrm{signal}$ \\
\hline $\begin{array}{c}\text { Energy Consumed by } \\
\left.\text { Transceiver's Circuitry( } \mathrm{E}_{\mathrm{elec}}\right)\end{array}$ & $50 \mathrm{~nJ} / \mathrm{bit}$ \\
\hline $\begin{array}{c}\text { Energy Expenditure in Transmit- } \\
\text { Amplification in free-space } \\
\left.\text { model( } \mathrm{e}_{\mathrm{fs}}\right)\end{array}$ & $10 \mathrm{pJ} / \mathrm{bit} / \mathrm{m}^{2}$ \\
\hline $\begin{array}{c}\text { Energy Expenditure Transmit- } \\
\text { Amplification in multipath fading } \\
\text { model }\left(\mathrm{E}_{\mathrm{mp}}\right)\end{array}$ & $0.0013 \mathrm{pJ} / \mathrm{bit} / \mathrm{m}^{4}$ \\
\hline
\end{tabular}

\subsubsection{Simulation Metric:}

The performance of the scheme is evaluated considering network lifetime as a parameter which is defined as the time until the last node dies in the network. Network lifetime is measured using following yard-stick.

- Number of nodes alive in the network $\rightarrow$ more number of nodes alive involves network lifetime last longer.

\subsubsection{Result and Discussion:}

A set of experiments is conducted to compare the performance of present scheme EACPLEP with $\mathrm{E}^{3} \mathrm{PSC}$ [11].

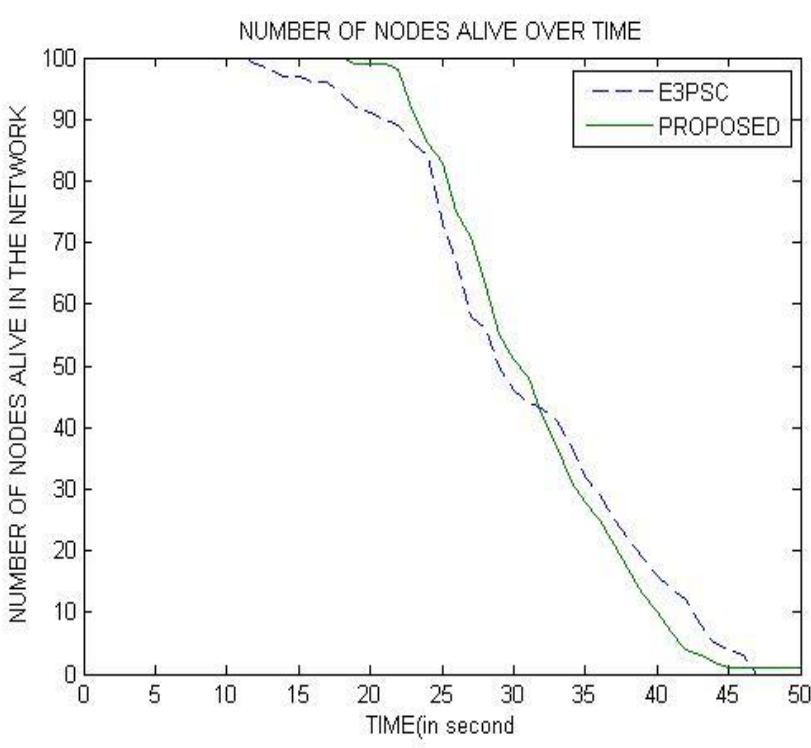

Figure 2. Number of nodes alive over time

Fig. 2 shows that the number of nodes alive in the network in EACPLEP is greater than the number of nodes alive in the $E^{3} P S C$. In EACPLEP occurrence of first node's death happens after $380 \mathrm{sec}$. but the same event in $E^{3} P S C$ occurs after $240 \mathrm{sec}$. $58.3 \%$ gain of EACPLEP over $\mathrm{E}^{3} \mathrm{PSC}$ in terms of number of nodes alive in the network.

\section{CONCLUSION}

In this paper an enhanced energy-efficient routing scheme EACPLEP for WSN is proposed. The EACPLEP is developed by altering an existing routing scheme $E^{3} P S C$ with an objective to prolong network lifetime further (more number of nodes alive in the network). Similar to $\mathrm{E}^{3} \mathrm{PSC}$, the present scheme divides network into a number of distance based static clusters. Though, in order to select cluster heads, it is based on density of number of nodes where the number of nodes are more, the cluster head formed that side, besides considering the $\mathrm{R}_{\text {energy }}$ and $\mathrm{L}_{\text {energy }}$ of individual nodes.

\section{FUTURE WORK}

As a future extension of this work, to achieve gain in terms of network lifetime, event-driven applications with heterogeneous energy model of sensor nodes may be investigated.

\section{REFERENCES}

[1] F. Zhao and L. Guibas, "Wireless Sensor Networks: An Information Processing Approach (Morgan Kaufmann Series in Networking)." San Mateo, CA: Morgan Kaufmann, 2004

[2] F. Akyildiz, Weilian Su, Yogesh Sankarasubramaniam, and Erdal Cayirci, "A Survey on Sensor Networks", IEEE Communication Magazine 40(8) 2002, pp. 393422 .

[3] J. N. Al-Karaki and A. E. Kamal, "Routing Techniques in Wireless Sensor Networks: A Survey”, IEEE Wireless Communication Vol.11, No.6, Dec.2004, pp. 6-28.

[4] Soheil Ghiasi, Ankur Srivastava, Xiaojian Yang, and Majid Sarrafzadeh, "Optimal Energy Aware Clustering in Sensor Networks", SENSORS Journal, Vol. 2, No. 7, 2002, pp. 258-269. 
[5] W. R. Heinzelman, A. Chandrakasan, and H. Balkrishnan, "Energy-Efficient Communication Protocol for Wireless Microsensor Networks", in Proceedings of 33rd Hawaii International Conference on System Science, Vol. 2, Jan. 2000, pp.1-10.

[6] W. R. Heinzelman A. Chandrakasan, and H.Balkrishnan, "An Application-Specific Protocol Architecture for Wireless Microsensor Networks", IEEE Trans. Wireless Communication, Vol. 1, No. 4, Oct. 2002, pp. 660-670.

[7] S. Lindsey, C. Raghvendra, and K. Shivlingam, “ Data Gathering in sensor network using the energy delay metric", in Proceedings of the IPDPS Workshop on issue in Wireless Sensor Network and Mobile Computing, San Fransisco, CA, USA, 2001, pp. 2001-2008.

[8] S. Lindsey, C. Raghvendra, and K. Shivlingam, "PEGASIS: Power-efficient gathering in sensor information System", in Proceedings of IEEE Aerospace Conference, Big Sky, MT, USA, 2002, pp. 1125- 1130.

[9] O. Younis and S. Fahmy, "HEED: A Hybrid EnergyEfficient Distributed Clustering Approach for Ad hoc Sensor Networks", IEEE Transaction on Mobile Computing, Vol. 3, No. 4, 2004, pp. 660-669.

[10] A.S. Zahmati, B.Abolhassani, Ali A.B.Shirazi, and A.S. Bahitiari, "An Energy-Efficient Protocol with Static Clustering for Wireless Sensor Networks", International Journal of Electronics, Circuit, and Systems Vol. 1, No. 2, May. 2007, pp. 135-138.

[11] S.K. Chaurasiya, Tumpa Pal, and Sipra Das Bit, “An Enhanced Energy-Efficient Protocol with static Clustering for WSN", IEEE,Vol. 3, No. 11,2011, pp. 658-663. 